\title{
Aldosterone is synthesized in and activates bulbospinal neurons through mineralocorticoid receptors and ENaCs in the RVLM
}

\author{
Naoki Oshima ${ }^{1}$, Hiroshi Onimaru ${ }^{2}$, Hanako Takechi ${ }^{1}$, Kojiro Yamamoto ${ }^{1}$, Atsushi Watanabe ${ }^{1}$, \\ Takahiro Uchida ${ }^{1}$, Yasuhiro Nishida ${ }^{3}$, Takashi Oda ${ }^{1}$ and Hiroo Kumagai ${ }^{1}$
}

The effects of aldosterone and mineralocorticoid receptor (MR) blockers on presympathetic neurons in the rostral ventrolateral medulla (RVLM) are well studied. To directly investigate whether aldosterone, eplerenone (an MR blocker), FAD286 (an aldosterone synthase inhibitor) and benzamil (an epithelial sodium channel (ENaC) blocker) affect RVLM neurons, we examined changes in the membrane potentials (MPs) of bulbospinal RVLM neurons using the whole-cell patch-clamp technique during superfusion with these drugs to brainstem-spinal cord preparations. Aldosterone superfusion $(0.1 \mu \mathrm{mol} / \mathrm{l})$ depolarized the RVLM neurons. In contrast, eplerenone superfusion $(1 \mu \mathrm{mol} / \mathrm{l})$ hyperpolarized them. To evaluate the existence of aldosterone, FAD286 superfusion $(10 \mu \mathrm{mol} / \mathrm{l})$ was performed, and the RVLM neurons became hyperpolarized during FAD superfusion. These data suggest that MRs exist and that aldosterone is synthesized in the brainstem. Benzamil superfusion $(1 \mu \mathrm{mol} / \mathrm{l})$ hyperpolarized the RVLM neurons. To clarify whether aldosterone, eplerenone, FAD286 and benzamil acted directly on the RVLM neurons, a low$\mathrm{Ca}^{2+}$, high-Mg ${ }^{2+}$ solution was used to block the synaptic input to the RVLM neurons, and the above-mentioned drugs were added during the low- $\mathrm{Ca}^{2}+$ superfusion. During the aldosterone superfusion, the RVLM neurons became depolarized, and they became hyperpolarized during eplerenone, FAD286 or benzamil superfusion. Importantly, when aldosterone was superfused after the benzamil solution, the MPs of the RVLM neurons did not depolarize. These results suggest that MRs are present in the RVLM neurons and that aldosterone is synthesized in the RVLM. The RVLM neurons themselves possess ENaCs, and ENaCs are the underlying mechanism by which aldosterone activates RVLM neurons.

Hypertension Research (2013) 36, 504-512; doi:10.1038/hr.2012.224; published online 31 January 2013

Keywords: aldosterone; benzamil; FAD286; RVLM neurons; whole-cell patch clamp

\section{INTRODUCTION}

The actions of aldosterone include vasoconstriction, vascular fibrosis, endothelial dysfunction, and sodium reabsorption and retention. These actions contribute to hypertension. However, recently, many studies have demonstrated the relationship between aldosterone and sympathetic nerve activity. In fact, aldosterone has been shown to cross the blood-brain barrier relatively easily, ${ }^{1}$ as clarified using the model developed by Gomez-Sanchez et al. ${ }^{2}$ Gomez-Sanchez et al. ${ }^{2}$ showed that the intracerebroventricular infusion of antagonists of the mineralocorticoid receptor (MR), at a dose that had no effect systemically, lowered blood pressure. Intracerebroventricular infusion of aldosterone increased blood pressure, ${ }^{3}$ and MRs were found in the brain. ${ }^{4}$ Furthermore, aldosterone is involved in remodeling after myocardial infarction, and central blockade of aldosterone has been shown to attenuate cardiac remodeling. 5,6

Recent studies have demonstrated the existence of an aldosterone system in the brain, ${ }^{4}$ and an increase in $\left[\mathrm{Na}^{+}\right]$in cerebrospinal fluid
(CSF) has been shown to increase aldosterone biosynthesis. ${ }^{7}$ Although the mechanism was unknown, Zhang et al..$^{3}$ demonstrated that aldosterone upregulated key elements (angiotensin II type 1 receptor and angiotensin-converting enzyme in the brain reninangiotensin system) and induced oxidative stress in the hypothalamus. They suggested that aldosterone increased sympathetic nerve activity through those mechanisms. In the brainstem, neurons that contain hydroxysteroid dehydrogenase 2 and MRs were present in the nucleus tractus solitarius. ${ }^{8}$ In the rostral ventrolateral medulla (RVLM), Zhu et al. ${ }^{9}$ and Nakagaki et al. ${ }^{10}$ reported that microinjection of aldosterone into the RVLM increased blood pressure.

However, only a few reports have discussed whether aldosterone directly activates bulbospinal RVLM neurons and whether MRs are present in RVLM neurons. Additionally, few reports have examined whether aldosterone is synthesized in the RVLM. Thus, in the present study, we investigated the effect of aldosterone, eplerenone (an MR

${ }^{1}$ Department of Nephrology and Endocrinology, National Defense Medical College, Tokorozawa, Japan; ²Department of Physiology, Showa University School of Medicine, Tokyo, Japan and ${ }^{3}$ Department of Physiology, National Defense Medical College, Tokorozawa, Japan

Correspondence: Dr N Oshima, Department of Nephrology and Endocrinology, National Defense Medical College, 3-2 Namiki, Tokorozawa, Saitama 359 8513, Japan.

E-mail: oshima@ndmc.ac.jp

Received 10 October 2012; revised 14 November 2012; accepted 15 November 2012; published online 31 January 2013 
blocker), FAD286 (an aldosterone synthase inhibitor) and benzamil (an epithelial sodium channel (ENaC) blocker) on bulbospinal RVLM neurons using brainstem-spinal cord preparations that preserved the sympathetic nervous system. ${ }^{11-20}$ As a previous report showed that intracerebroventricular infusion of eplerenone lowered blood pressure, ${ }^{2,21}$ we examined the direct effect of eplerenone on RVLM neurons, using a low- $\mathrm{Ca}^{2+}$, high- $\mathrm{Mg}^{2+}$ solution to block synaptic input into RVLM neurons. Aldosterone performs its function in the collecting ducts of the kidneys, and ENaCs are known to participate in the process through genomic action. As ENaCs were shown to exist in the choroid plexus, ${ }^{22}$ we investigated how quickly aldosterone activated bulbospinal RVLM neurons and whether ENaCs were involved in the response caused by aldosterone.

\section{METHODS}

\section{General preparations}

Experiments were performed on the brainstem-spinal cord preparations collected from 1-5-day-old Wistar rats, as previously described. ${ }^{12-20}$ The experimental protocols were approved by the Institutional Review Board of National Defense Medical College and were in accordance with the National Guidelines for the Conduct of Animal Experiments. Briefly, the animals received deep ether anesthesia, and the brainstem-spinal cord was isolated at the $\mathrm{Th}_{2}$ level (Figure 1a). The brainstem was sectioned between the roots of cranial nerve VI and the lower border of the trapezoid body. The preparation was continuously superfused with a solution containing in the following solutes in mmoll-1: $124 \mathrm{NaCl}, 5.0 \mathrm{KCl}, 1.2 \mathrm{KH}_{2} \mathrm{PO}_{4}$, $2.4 \mathrm{CaCl}_{2}, 1.3 \mathrm{MgCl}_{2}, 26 \mathrm{NaHCO}_{3}$ and 30 glucose. The preparation was maintained at $25-26^{\circ} \mathrm{C}$ (artificial CSF (aCSF)). The $\mathrm{pH}(7.4)$ and oxygenation were maintained by bubbling $90 \% \mathrm{O}_{2}-5 \% \mathrm{~N}_{2}-5 \% \mathrm{CO}_{2}$ through the solution. To avoid recording respiratory neuronal activity, we examined phrenic nerve activity from the ventral root of $\mathrm{C}_{4}$ (Figure 1a) and excluded neurons with a strong phase relationship with the phrenic nerve discharge from further study. ${ }^{12,16,17}$

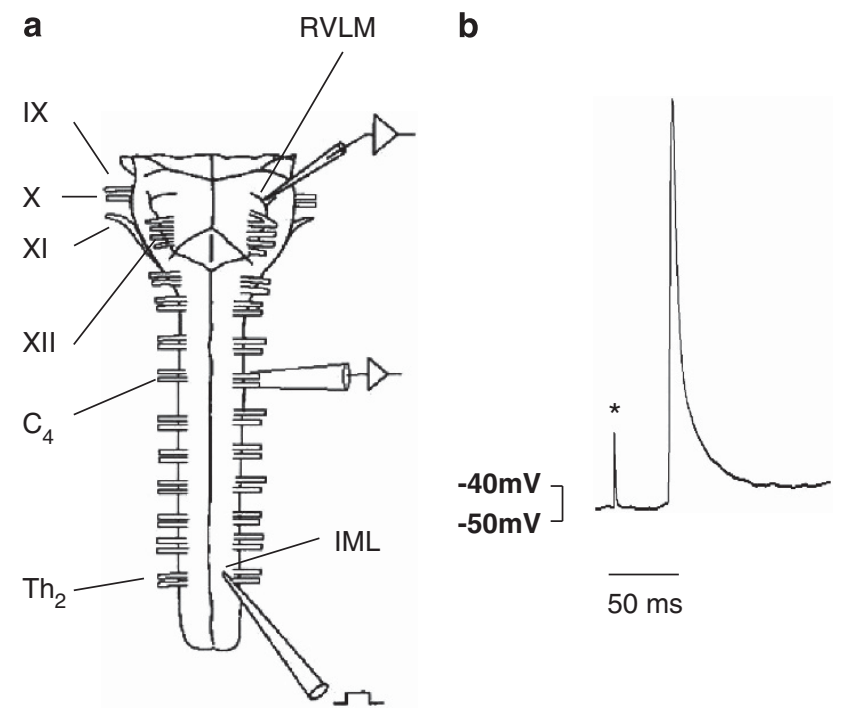

Figure 1 (a) The brainstem-spinal cord preparation of a neonatal Wistar rat. $I X, X, X I$ and $X I I$ are the cranial nerves. Phrenic nerve activity was examined in the ventral root of $C_{4}$. Electrical stimulation was applied to the intermediolateral cell column at the $\mathrm{Th}_{2}$ level via an electrode. (b) An antidromic AP in a RVLM neuron that was evoked by electrical stimulation ${ }^{*}$ ) in the intermediolateral cell column at the level of $\mathrm{Th}_{2}$.

\section{Patch-clamp electrodes}

Electrodes were pulled in one stage from thin-wall borosilicate filament capillaries (GC100TF-10, outer diameter $1.0 \mathrm{~mm}$, Clark Electromed, Reading, UK) using a vertical puller. The electrodes had a tip diameter of $1.8-2.0 \mu \mathrm{m}$ and a resistance of $4-6 \mathrm{M} \Omega$. The electrode solution for the whole-cell recording consisted of the following in mmol/l: 130 potassium gluconate, $10 \mathrm{HEPES}, 10 \mathrm{EGTA}, 1 \mathrm{CaCl}_{2}$ and 1 $\mathrm{MgCl}_{2}$, with $\mathrm{pH} 7.2-7.3$ adjusted using $\mathrm{KOH}$. The electrode tips were filled with $1 \%$ Lucifer-Yellow (Sigma, St Louis, MO, USA).

\section{Recording procedure}

Electrodes were inserted into the RVLM. A patch-clamp amplifier (AxoPatch, ID; Axon Instruments, Sunnyvale, CA, USA) was used to record the membrane potentials (MPs). The RVLM neurons were found on the ventral side of the medulla. Before making the intracellular whole-cell recordings, we observed the firing pattern of the target neurons using extracellular recordings. After obtaining a G $\Omega$ seal, a single-shot hyperpolarizing pulse (0.6-0.9 nA; duration, $30 \mathrm{~ms}$ ) was applied to rupture the neuronal membrane. To determine whether each recorded RVLM neuron was a bulbospinal one, the existence of antidromic action potentials (APs) in a RVLM neuron (Figure 1b) was examined by electrical stimulation of the intermediolateral cell column at the $\mathrm{Th}_{2}$ level using a tungsten electrode ( $30 \mu \mathrm{m}$ tip diameter; Unique Medical, Tokyo, Japan). The RVLM neurons that had antidromic APs with electrical stimulation in the intermediolateral cell column at the $\mathrm{Th}_{2}$ level were considered to be bulbospinal RVLM neurons. The MPs were recorded using the current-clamp technique $(20 \mathrm{pA}$ increments from $-100-20 \mathrm{pA}, 500 \mathrm{~ms}$ in duration). All of the data were recorded and analyzed using PowerLab (AD Instruments, Colorado Springs, CO, USA). The membrane resistances for the RVLM neurons were calculated from current-voltage curves. During the course of the whole-cell recordings, the neurons were labeled with Lucifer-Yellow either by spontaneous diffusion or iontophoresis. A chloride ion equilibrium potential of $-89 \mathrm{mV}$ was calculated using the Nernst equation and the intracellular and extracellular chloride concentrations.

\section{Experimental protocols}

Protocol (1). The bulbospinal RVLM neurons were superfused with each drug. The duration of each drug (except FAD286) superfusion ranged from 10-15 min. During superfusion with each drug, we defined depolarization as an increase of more than $2 \mathrm{mV}$ in the MP and hyperpolarization as a decrease of more than $2 \mathrm{mV}$ in the MP. The change in the MPs was determined $10 \mathrm{~min}$ after starting superfusion with each drug.

The duration of FAD286 superfusion was $5 \mathrm{~min}$. The definitions of depolarization and hyperpolarization were the same as those described above. The change in the MPs was determined at the points where the minimal or maximum values were recorded after stopping superfusion with FAD286 because the effect of FAD286 was maintained even after FAD286 superfusion was stopped.

The changes in the excitatory post-synaptic potentials (EPSPs) found during drug superfusion were assessed by predefined scores. In a 2-min period, a change of more than $4 \mathrm{mV}$ in the EPSP was assessed as one point and a change of more than $6 \mathrm{mV}$ as two points. Summed points before drug superfusion (baseline) were compared with those during drug superfusion. If the result was less than one-half or more than twice the baseline, we defined the difference as positive. The change in the EPSPs was determined 9-10 min after starting superfusion with each drug.

Protocol (2). During MP recordings of the bulbospinal RVLM neurons, the preparations were superfused with aldosterone 
$\left(0.1 \mu \mathrm{moll}{ }^{-1},{ }^{23}\right.$ Sigma $)$, eplerenone $\left(1 \mu \mathrm{moll}^{-1},{ }^{23}\right.$ Sigma $)$, FAD286 $\left(10 \mu \mathrm{moll} 1^{-1},{ }^{24}\right.$ Novartis Institutes for BioMedical Research) and benzamil ( $1 \mu \mathrm{moll} 1^{-1},{ }^{25,26}$ Sigma $)$ dissolved in aCSF.

Protocol (3). The MPs for the bulbospinal RVLM neurons were recorded during superfusion with a low- $\mathrm{Ca}^{2+}$, high- $\mathrm{Mg}^{2+}$ solution that included aldosterone, eplerenone, FAD286 or benzamil. Before each drug superfusion, a low- $\mathrm{Ca}^{2+}$, high- $\mathrm{Mg}^{2+}$ superfusion was performed for over 15 min to block synaptic transmission from other neurons to the recorded bulbospinal RVLM neuron. ${ }^{27}$

\section{Histology}

At the conclusion of each experiment, the preparations were fixed for at least $48 \mathrm{~h}$ at $4{ }^{\circ} \mathrm{C}$ in phosphate-buffered $10 \%$ formalin, and transverse $100-\mu \mathrm{m}$ frozen sections were cut with a custom-made vibratome. Lucifer-Yellow-labeled RVLM neurons were photographed using a fluorescence microscope (BH-2; Olympus, Tokyo, Japan). Selected neurons were traced with a camera lucida attachment at a magnification of $\times 400 .{ }^{12,19,20}$ Neutral-Red staining was performed to verify the position of the neurons within the RVLM, and the precise location of the marked neurons was identified.

\section{Statistics}

The results are expressed as the means \pm s.e.m. Comparisons of the MPs before and during drug superfusion were evaluated using the Student's $t$-test for paired observations. Statistical significance was set at $P<0.05$.

\section{RESULTS}

The recorded bulbospinal RVLM neurons $(n=90)$ were classified into four types based on their firing patterns: three types that we previously reported ${ }^{12}$ (regularly firing neurons $(n=10$; resting membrane potential, $-41.9 \pm 0.1 \mathrm{mV}$; frequency of APs (frequency of APs), $3.7 \pm 0.2$ spikes per second), irregularly firing neurons $\left(n=52\right.$; resting MP, $-44.3 \pm 0.9 \mathrm{mV}^{\#}$; frequency of APs, $1.0 \pm 0.2$ spikes per second) ( ${ }^{\#} P<0.05$ vs. regularly firing neurons), and silenttype neurons $\left(n=16\right.$; resting MP, $\left.-49.9 \pm 1.5 \mathrm{mV}^{\#}\right)\left({ }^{\#} P<0.05\right.$ vs. regularly firing neurons)). The RVLM contained significant amounts of burst-type neurons $\left(n=12\right.$; resting MP, $-47.0 \pm 0.8 \mathrm{mV}^{\star}$; AP: $1.4 \pm 0.3$ spikes per second $)\left({ }^{\star} P<0.01\right.$ vs. regularly firing neurons $)$, which were newly found in this study (Figures $2 \mathrm{~A}, \mathrm{~B}$ and $3 \mathrm{~A}, \mathrm{~B}$ ).

In our previous report, ${ }^{12}$ we classified bulbospinal RVLM neurons into three types: regularly firing neurons, irregularly firing neurons and silent-type neurons. Regularly firing neurons exhibited frequent APs ( $3.7 \pm 0.2$ spikes per second) with rare EPSPs. The MP trajectory of these neurons consisted of a gradual depolarizing interspike ramp, resulting in an AP and after-polarization. The EPSPs did not evoke APs. The irregularly firing neurons exhibited a lower frequency of APs $(1.0 \pm 0.2$ spikes per second) with numerous PSPs. Some of the EPSPs evoked APs. The silent-type neurons exhibited APs only during current-induced depolarization. In the present study, we identified a fourth type of neuron, the bulbospinal RVLM, which exhibited a firing pattern that included bursts. The recordings from these bursttype neurons exhibited a relatively irregular burst pattern (Figures 2A, $\mathrm{B}$ and $3 \mathrm{~A}, \mathrm{~B}, \mathrm{D})$.

Of the 19 neurons that were examined (regularly firing, 1; irregularly firing, 10; silent-type, 2 and burst-type, 6), depolarization during aldosterone superfusion was observed in 14 (regularly firing, 1; irregularly firing, 6; silent-type, 2 and burst-type, 5). Regarding the membrane resistances of the bulbospinal RVLM neurons, there were no significant differences between before and during each drug superfusion (Table 1A).
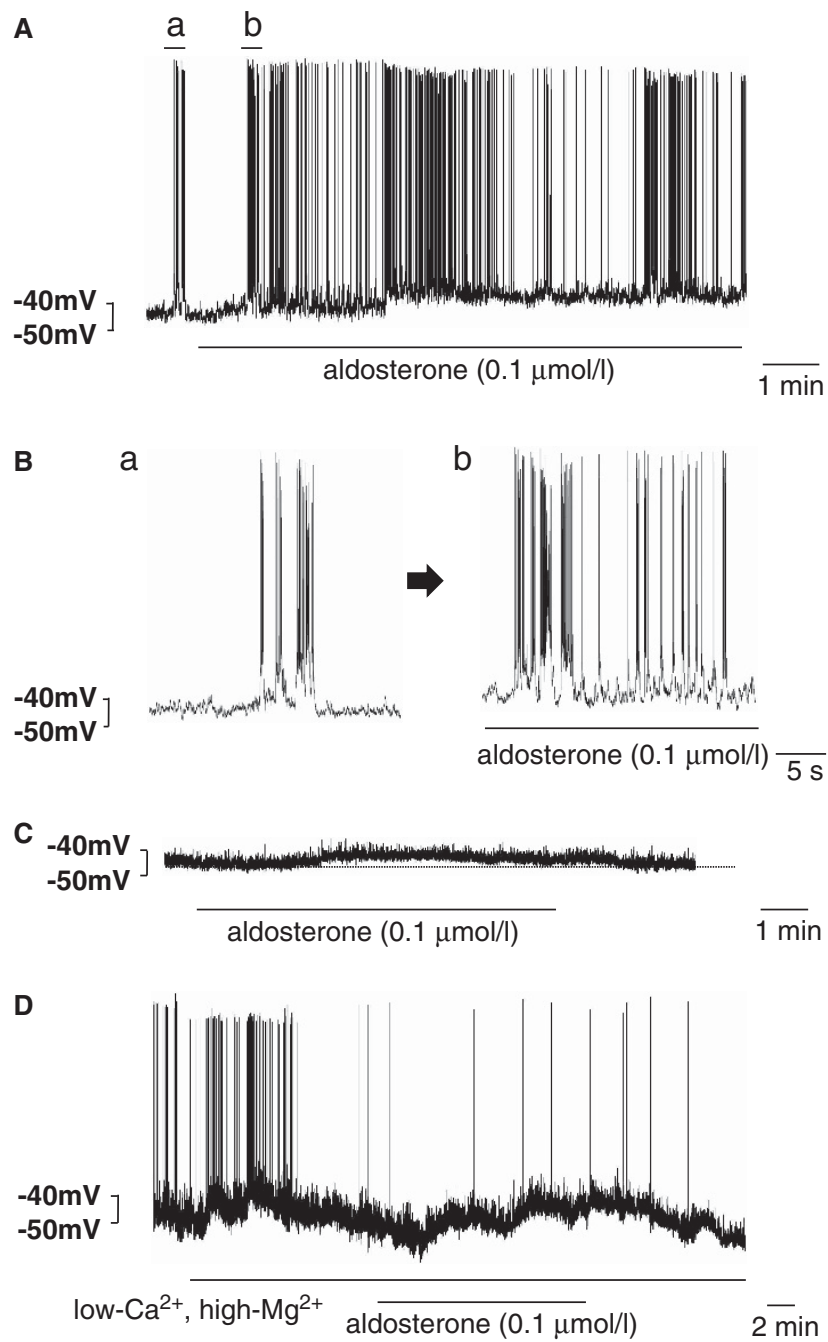

Figure 2 Depolarization of a bulbospinal RVLM neuron during aldosterone superfusion. (A) After starting aldosterone superfusion, the neuron depolarized and had strong EPSP activity within $2 \mathrm{~min}$. In this neuron, the MPs before (a) and after (b) starting aldosterone superfusion are indicated in Figure 2b. (B) The amplitude of the bulbospinal RVLM neuron EPSPs increased during aldosterone superfusion (b) compared to that before aldosterone superfusion (a). This neuron had a burst-type AP. (C) During aldosterone superfusion, the MP of the neuron depolarized. This neuron was a silent-type one and had no APS. (D) Depolarization in a bulbospinal RVLM neuron during superfusion with aldosterone dissolved in a low-Ca ${ }^{2+}$, high$\mathrm{Mg}^{2}+$ solution. This result suggests that the RVLM neuron was spontaneously excited during aldosterone superfusion without input from other neurons.

\section{Effect of aldosterone on bulbospinal RVLM neurons}

Fourteen of the 19 bulbospinal RVLM neurons depolarized during aldosterone superfusion (Figures $2 \mathrm{~A}$ and C, Table 1A). All of these 14 neurons depolarized within a few minutes after starting aldosterone superfusion. Ten of the 14 depolarized RVLM neurons showed increased EPSP amplitudes and frequencies (before, $11.3 \pm 3.8$ points per minute; during, $32.7 \pm 9.5$ points per minute $\left.{ }^{\#}\right)\left({ }^{\#} P<0.05\right.$ vs. before aldosterone superfusion) (Figure 2B). Three of the 19 bulbospinal RVLM neurons showed hyperpolarization during aldosterone superfusion (Table 1A). Two out of these three hyperpolarized RVLM neurons showed increased EPSP amplitudes and frequencies (before, 
A

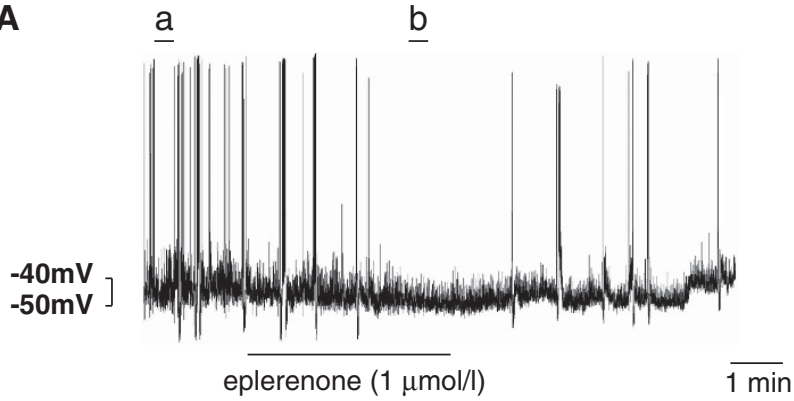

B
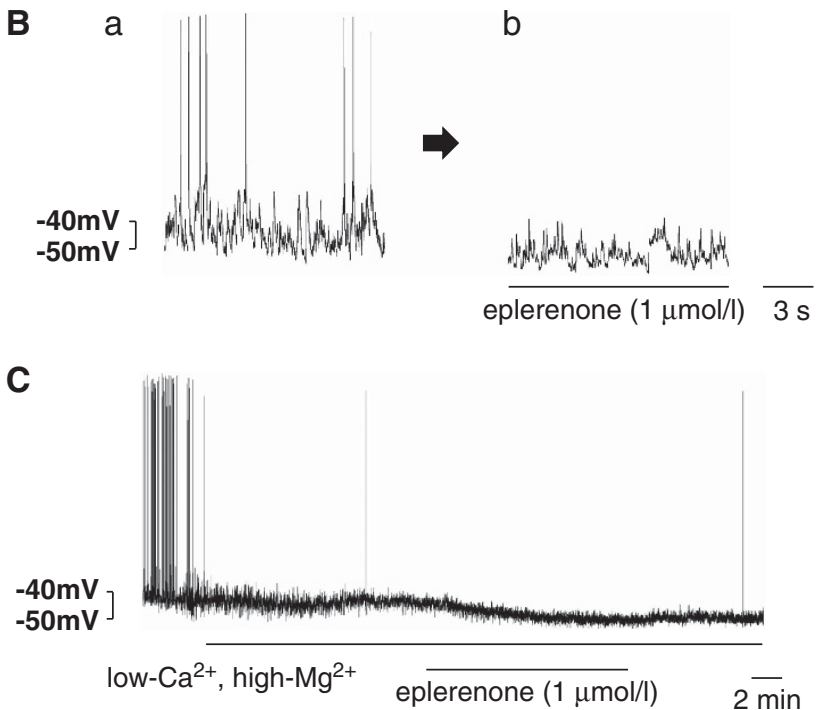

D

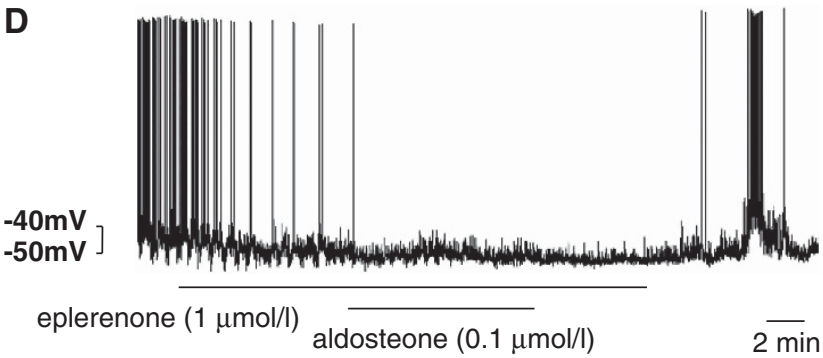

Figure 3 Hyperpolarization of a bulbospinal RVLM neuron during eplerenone superfusion. (A) Hyperpolarization and decreased AP frequency in the bulbospinal RVLM neurons were shown during eplerenone superfusion. In this neuron, the MPs before (a) and after (b) starting eplerenone superfusion are indicated in Figure 4b. (B) The EPSP amplitudes in the bulbospinal RVLM neuron were smaller during eplerenone superfusion (b) than before eplerenone superfusion (a). This neuron had a burst-type AP. (C) Hyperpolarization of a RVLM neuron during superfusion with eplerenone dissolved in a low- $\mathrm{Ca}^{2+}$, high- $\mathrm{Mg}^{2+}$ solution. This result suggests that the bulbospinal RVLM neuron by itself has MRs without affecting the synaptic input from other neurons. (D) Attenuated effect of aldosterone on an RVLM neuron during eplerenone superfusion. During superfusion with eplerenone, the addition of aldosterone did not depolarize the RVLM neuron. In this figure, a slight increase in the MP $(+1 \mathrm{mV})$ was observed during aldosterone superfusion.

$4.5 \pm 0.5$ points per minute; during, $27.0 \pm 13.0$ points per minute) (Figure 4B). These EPSPs suggest that other neurons and astrocytes send inputs to the RVLM neurons and excite them.

To block the effects of such inputs, we superfused the preparation with a low- $\mathrm{Ca}^{2+}$, high- $\mathrm{Mg}^{2+}$ solution. Nine out of the 14
Table 1A Changes in MPs of bulbospinal RVLM neurons during superfusion with each drug

\begin{tabular}{|c|c|c|}
\hline Drug & Membrane potential (mV) & Membrane resistance $(M \Omega)$ \\
\hline \multicolumn{3}{|l|}{ Aldosterone } \\
\hline \multicolumn{3}{|c|}{ Depolarization $(\mathrm{n}=14)$} \\
\hline Before & $-48.0 \pm 1.3$ & $221 \pm 35$ \\
\hline After & $-43.9 \pm 1.1^{*}$ & $217 \pm 30$ \\
\hline \multicolumn{3}{|c|}{ No change $(\mathrm{n}=2)$} \\
\hline Before & $-46.0 \pm 2.9$ & $612 \pm 124$ \\
\hline After & $-47.1 \pm 2.1$ & $513 \pm 75$ \\
\hline \multicolumn{3}{|c|}{ Hyperpolarization $(\mathrm{n}=3)$} \\
\hline Before & $-48.9 \pm 1.9$ & $469 \pm 135$ \\
\hline After & $-52.4 \pm 2.1^{\#}$ & $488 \pm 104$ \\
\hline \multicolumn{3}{|l|}{ Eplerenone } \\
\hline \multicolumn{3}{|c|}{ Depolarization $(\mathrm{n}=2)$} \\
\hline Before & $-44.8 \pm 0.8$ & $759 \pm 25$ \\
\hline After & $-40.3 \pm 1.4^{\#}$ & $738 \pm 253$ \\
\hline \multicolumn{3}{|c|}{ No change $(n=0)$} \\
\hline \multicolumn{3}{|c|}{ Hyperpolarization $(\mathrm{n}=13)$} \\
\hline Before & $-44.0 \pm 1.1$ & $440 \pm 103$ \\
\hline After & $-48.1 \pm 1.4^{\#}$ & $532 \pm 142$ \\
\hline \multicolumn{3}{|l|}{ FAD286 } \\
\hline \multicolumn{3}{|c|}{ Depolarization $(n=0)$} \\
\hline \multicolumn{3}{|c|}{ No change $(n=0)$} \\
\hline \multicolumn{3}{|c|}{ Hyperpolarization $(\mathrm{n}=5)$} \\
\hline Before & $-40.4 \pm 0.5$ & $602 \pm 191$ \\
\hline After & $-47.5 \pm 1.4^{\#}$ & $524 \pm 145$ \\
\hline \multicolumn{3}{|l|}{ Benzamil } \\
\hline \multicolumn{3}{|c|}{ Depolarization $(\mathrm{n}=1)$} \\
\hline Before & -48.0 & 334 \\
\hline After & -43.5 & 322 \\
\hline \multicolumn{3}{|c|}{ No change $(\mathrm{n}=3)$} \\
\hline Before & $-44.7 \pm 2.7$ & $375 \pm 184$ \\
\hline After & $-44.5 \pm 2.5$ & $369 \pm 177$ \\
\hline \multicolumn{3}{|c|}{ Hyperpolarization $(\mathrm{n}=14)$} \\
\hline Before & $-45.4 \pm 1.2$ & $350 \pm 43$ \\
\hline After & $-49.9 \pm 1.2^{*}$ & $328 \pm 35$ \\
\hline
\end{tabular}

Values are mean \pm s.e.m. ${ }^{*} P<0.01,{ }^{\#} P<0.05$ vs before each drug superfusion.

bulbospinal RVLM neurons depolarized during superfusion of aldosterone dissolved in a low- $\mathrm{Ca}^{2+}$, high- $\mathrm{Mg}^{2+}$ solution (Figure 2D, Table 1B). The results suggest that these neurons had depolarized by themselves without receiving input from other neurons.

Effect of eplerenone on bulbospinal RVLM neurons

Thirteen of the 15 bulbospinal RVLM neurons became hyperpolarized during eplerenone superfusion (Figure 3A, Table 1A). Among these 13 hyperpolarized RVLM neurons, six neurons showed decreased EPSP amplitudes and frequencies (before, $32.1 \pm 11.0$ points per minute; during, $13.1 \pm 6.0$ points per minute $\left.{ }^{\#}\right)\left({ }^{\#} P<0.05\right.$ vs. before eplerenone superfusion) (Figure $3 \mathrm{~B}$ ).

Eight of the 10 bulbospinal RVLM neurons became hyperpolarized during superfusion with eplerenone dissolved in a low- $\mathrm{Ca}^{2+}$, high$\mathrm{Mg}^{2+}$ solution (Figure 3C, Table 1B). These results strongly suggested the existence of MRs in the bulbospinal RVLM neurons. 
Table 1B Changes in MPs of bulbospinal RVLM neurons during superfusion with each drug dissolved in a low- $\mathrm{Ca}^{2+}$, high- $\mathrm{Mg}^{2+}$ solution

\begin{tabular}{|c|c|}
\hline Drug & Membrane potential ( $\mathrm{mV}$ ) \\
\hline \multicolumn{2}{|l|}{ Aldosterone } \\
\hline \multicolumn{2}{|c|}{ Depolarization $(n=9)$} \\
\hline Before & $-51.2 \pm 2.6$ \\
\hline After & $-44.9 \pm 1.8^{\#}$ \\
\hline \multicolumn{2}{|c|}{ No change $(n=1)$} \\
\hline Before & -40.6 \\
\hline After & -41.0 \\
\hline \multicolumn{2}{|c|}{ Hyperpolarization $(n=4)$} \\
\hline Before & $-54.2 \pm 1.9$ \\
\hline After & $-59.2 \pm 2.0^{*}$ \\
\hline \multicolumn{2}{|l|}{ Eplerenone } \\
\hline \multicolumn{2}{|c|}{ Depolarization $(n=0)$} \\
\hline \multicolumn{2}{|c|}{ No change $(n=2)$} \\
\hline Before & $-41.5 \pm 1.9$ \\
\hline After & $-41.2 \pm 2.6$ \\
\hline \multicolumn{2}{|c|}{ Hyperpolarization $(\mathrm{n}=8)$} \\
\hline Before & $-47.7 \pm 3.2$ \\
\hline After & $-51.4 \pm 4.0^{\#}$ \\
\hline \multicolumn{2}{|l|}{ FAD286 } \\
\hline \multicolumn{2}{|c|}{ Depolarization $(n=0)$} \\
\hline \multicolumn{2}{|c|}{ No change $(n=0)$} \\
\hline \multicolumn{2}{|c|}{ Hyperpolarization $(n=4)$} \\
\hline Before & $-42.1 \pm 2.9$ \\
\hline After & $-47.8 \pm 2.8^{*}$ \\
\hline \multicolumn{2}{|l|}{ Benzamil } \\
\hline \multicolumn{2}{|c|}{ Depolarization $(n=0)$} \\
\hline \multicolumn{2}{|c|}{ No change $(n=0)$} \\
\hline \multicolumn{2}{|c|}{ Hyperpolarization $(n=7)$} \\
\hline Before & $-44.2 \pm 1.7$ \\
\hline After & $-46.8 \pm 1.3^{\#}$ \\
\hline
\end{tabular}

${ }^{*} P<0.01,{ }^{\#} P<0.05$ vs before superfusion with aldosterone dissolved in a low- $\mathrm{Ca}^{2+}$, high-Mg2+ solution.

In the 4 neurons that exhibited hyperpolarization during eplerenone superfusion (before, $-41.6 \pm 0.8 \mathrm{mV}$; during, $-46.0 \pm$ $\left.1.5 \mathrm{mV}^{\#}\right) \quad\left({ }^{\#} P<0.05\right.$ vs. before eplerenone superfusion), aldosterone was added to the superfused solution to examine the MR-blocking effect of eplerenone. As a result, all four bulbospinal RVLM neurons did not show any obvious changes during superfusion with aldosterone (before, $-46.0 \pm 1.5 \mathrm{mV}$; during, $-44.8 \pm 1.8 \mathrm{mV}^{\#}$ ) (Figure 3D). These results suggest that prior superfusion with eplerenone suppressed the effect of aldosterone on RVLM neurons.

To investigate whether eplerenone induced a reverse effect on RVLM neurons relative to aldosterone, eplerenone superfusion was applied after finishing the aldosterone superfusion. We examined the two bulbospinal RVLM neurons that showed hyperpolarization during aldosterone superfusion. In both cases, the RVLM neuron became hyperpolarized during aldosterone superfusion (Figure 4A, left panel), and the MPs returned to the resting level during a wash out with aCSF superfusion. After that, these neurons were depolarized during eplerenone superfusion (Figure 4A, right panel). Eplerenone and aldosterone thus had opposite effects.

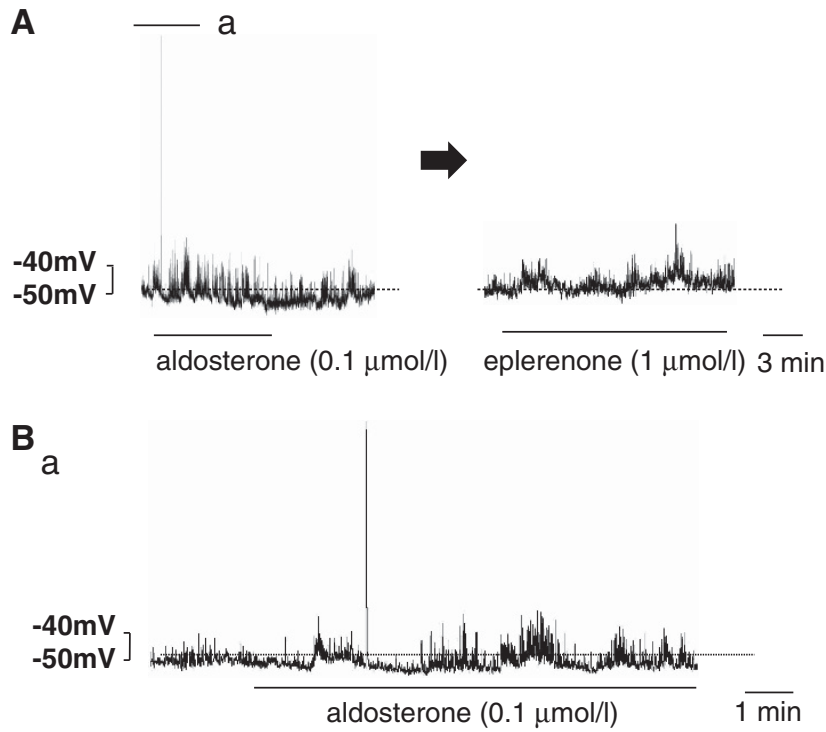

Figure 4 Eplerenone and aldosterone showed opposite effects. (A) A bulbospinal RVLM neuron hyperpolarized during aldosterone superfusion and returned to the resting level during subsequent aCSF superfusion (left panel). However, in this neuron, EPSP amplitudes increased during aldosterone superfusion (a). After 10 min of aCSF superfusion, the neuron was superfused with eplerenone. During eplerenone superfusion, the neuron depolarized (right panel). (B) Although during aldosterone superfusion the RVLM neuron was hyperpolarized, many high amplitude EPSPs appeared on the MPs of the neuron.

\section{Effect of FAD286 on bulbospinal RVLM neurons}

All five bulbospinal RVLM neurons became hyperpolarized (before, $-40.4 \pm 0.5 \mathrm{mV}$; after, $\left.-47.5 \pm 1.4 \mathrm{mV}^{\#}\right)\left({ }^{\#} P<0.05\right.$ vs. before FAD286 superfusion) during and after FAD286 superfusion (Figures $5 \mathrm{Aa}, \mathrm{b}$ and $\mathrm{B}$, and Table 1A). After starting the FAD286 superfusion, the RVLM neurons became hyperpolarized within $5 \mathrm{~min}$, and the effect was sustained (decreased MPs) for $\sim 10-20 \mathrm{~min}$ after stopping FAD286 superfusion (Figure 5B). All five hyperpolarized RVLM neurons had decreased EPSP amplitudes and frequencies (before, $27.8 \pm 8.4$ points per minute; after, $3.6 \pm 1.2$ points per minute $\left.{ }^{\#}\right)$ ( ${ }^{\#} P<0.05$ vs. before FAD286 superfusion) during and after FAD286 superfusion (Figure 5B).

All four bulbospinal RVLM neurons became hyperpolarized during and after superfusion with FAD286 dissolved in a low-Ca ${ }^{2+}$, high$\mathrm{Mg}^{2+}$ solution (before, $-42.1 \pm 2.9 \mathrm{mV}$; after, $-47.8 \pm 2.8 \mathrm{mV}^{\star}$ ) $\left({ }^{\star} P<0.01\right.$ vs. before FAD286 superfusion) (Figure $5 \mathrm{C}$, Table $\left.1 \mathrm{~B}\right)$. The effect of FAD286 was maintained for $\sim 5-10$ min after completing a FAD286 superfusion. These results suggest that aldosterone is synthesized in the RVLM.

\section{Effect of benzamil on bulbospinal RVLM neurons}

Fourteen of the 18 neurons became hyperpolarized during benzamil superfusion (Figure 6a, Table 1A). Among these 14 hyperpolarized RVLM neurons, 10 neurons showed decreased EPSP amplitudes and frequencies (before, $17.0 \pm 4.7$ points per minute; during, $5.1 \pm 2.2$ points per minute $\left.{ }^{\#}\right)\left({ }^{\#} P<0.05\right.$ vs. before benzamil superfusion) (Figure 6a). During superfusion with benzamil dissolved in a low$\mathrm{Ca}^{2+}$, high- $\mathrm{Mg}^{2+}$ solution, all of the seven bulbospinal RVLM neurons that were tested became hyperpolarized (Figure 6b, Table 1B). These results suggest the existence of ENaCs in the bulbospinal RVLM neurons. 


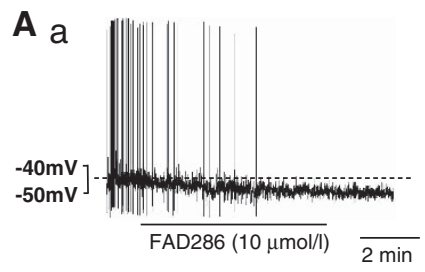

b

B

b

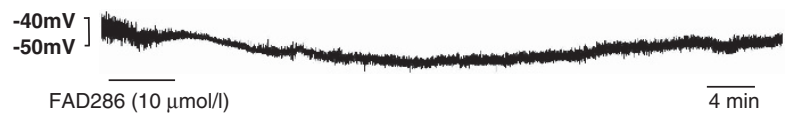

C

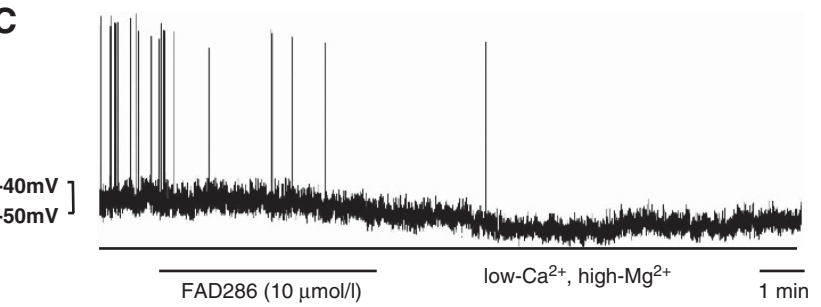

Figure 5 Hyperpolarization of the bulbospinal RVLM neurons during and after FAD286 (an aldosterone synthase inhibitor) superfusion. (A) (a) In an irregularly firing neuron, the MP was hyperpolarized, and the AP frequency decreased within 4 min after starting the FAD286 superfusion. (b) In a silent-type neuron, the MP was hyperpolarized, and decreased EPSP amplitudes and frequencies were observed after starting the FAD286 superfusion. (B) Long-term recording of the MP. The effect of FAD286 was sustained for about $20 \mathrm{~min}$ after cessation of the FAD286 superfusion. (C) The RVLM neuron became hyperpolarized during and after superfusion with FAD286 dissolved in a low- $\mathrm{Ca}^{2+}$, high- $\mathrm{Mg}^{2}+$ solution. Superfusion with a low- $\mathrm{Ca}^{2+}$, high- $\mathrm{Mg}^{2+}$ solution was performed for about $20 \mathrm{~min}$ before FAD286 superfusion. The effect of FAD286 was sustained for about 5 min after cessation of the FAD286 superfusion.

In three of these seven neurons, aldosterone was added to the solution, and all three bulbospinal RVLM neurons showed no change during superfusion with aldosterone (Figure $6 \mathrm{~b}$ ). The results suggest that ENaCs in the RVLM neurons were responsible for aldosteroneinduced RVLM activation.

\section{Histology}

Lucifer-Yellow staining was performed after whole-cell recordings of the bulbospinal RVLM neurons had been completed (Figures 7a-c). Ten recorded bulbospinal RVLM neurons were histologically examined, and their locations were plotted (Figure 7a). The average long axis of the soma of the RVLM neurons $(n=10$ : two regularly firing neurons, four irregularly firing neurons, two silent-type neurons and two burst-type neurons) measured $25.9 \pm 1.8 \mu \mathrm{m}$, and the number of primary dendrites was $5.0 \pm 0.8$. Lucifer-yellow staining showed that those neurons were all located in the RVLM.

\section{DISCUSSION}

In this study, we found that aldosterone depolarized and eplerenone (an MR blocker) hyperpolarized the bulbospinal RVLM neurons directly. Benzamil (an ENaC blocker) hyperpolarized the bulbospinal RVLM neurons directly, and aldosterone showed no effects during superfusion with benzamil dissolved in a low- $\mathrm{Ca}^{2+}$, high- $\mathrm{Mg}^{2+}$
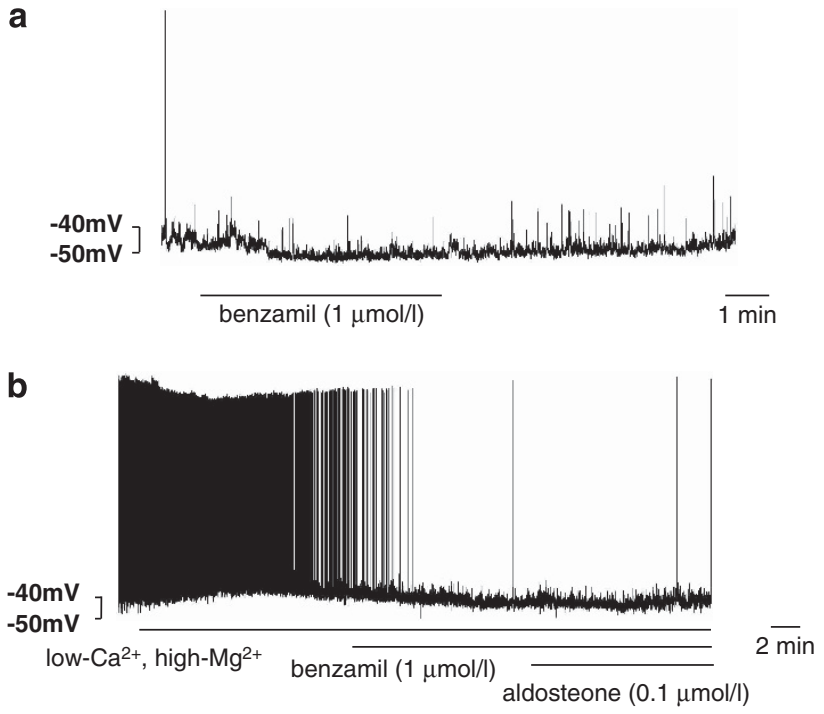

Figure 6 Hyperpolarization and a decreased EPSP amplitudes in a bulbospinal RVLM neuron during benzamil superfusion. (a) A bulbospinal RVLM neuron hyperpolarized during benzamil superfusion. This neuron had decreased EPSP amplitudes during benzamil superfusion. (b) Hyperpolarization in the bulbospinal RVLM neuron during superfusion with benzamil dissolved in a low- $\mathrm{Ca}^{2+}$, high- $\mathrm{Mg}^{2+}$ solution. During superfusion with benzamil, aldosterone did not induce RVLM neuronal depolarization.

solution. Thus, we considered that RVLM neurons themselves have MRs and ENaCs, and ENaCs are an underlying mechanism by which aldosterone activates RVLM neurons.

\section{Effect of aldosterone on bulbospinal neurons in the RVLM}

There have been some previous studies on aldosterone in the brainstem. Zhu et al. ${ }^{9}$ and Nakagaki et al. ${ }^{10}$ demonstrated that microinjected corticoids into the RVLM increased blood pressure, and Nakagaki et al. ${ }^{10}$ also reported that microinjected aldosterone increased blood pressure more in SHRSPs than Wistar rats. Furthermore, Kumar et al. ${ }^{4}$ detected aldosterone synthetase in ventrolateral medulla oblongata neurons, which regulate blood pressure.

However, direct evidence that aldosterone activates bulbospinal RVLM neurons, which mainly control blood pressure in the brain, has not been shown. Therefore, we examined whether aldosterone directly depolarizes RVLM neurons.

In 14 of the 19 bulbospinal RVLM neurons, aldosterone induced depolarization (Figures $2 \mathrm{a}$ and $\mathrm{c}$, Table 1A). These results may indicate that aldosterone increases sympathetic nerve activity by exciting bulbospinal RVLM neurons.

During superfusion with aldosterone, an increase in the amplitude of EPSPs was shown in 10 of the 14 depolarized RVLM neurons (Figure 2b). These results suggest that neurons other than the caudal ventrolateral medulla (CVLM) neurons sent excitatory inputs to the bulbospinal RVLM neurons. If the neurons that sent activated signals to the bulbospinal RVLM neurons had been CVLM neurons, the PSPs would have been inhibitory ones. Increased EPSPs amplitude was also shown in two of the three hyperpolarized RVLM neurons (Figure 4b). The results suggest that although recorded RVLM neurons received excitatory inputs from other neurons, RVLM neurons themselves were hyperpolarized by aldosterone.

Therefore, to negate the influence of neurons sending signals to the RVLM neurons, we used a low- $\mathrm{Ca}^{2+}$, high- $\mathrm{Mg}^{2+}$ solution. Nine of 


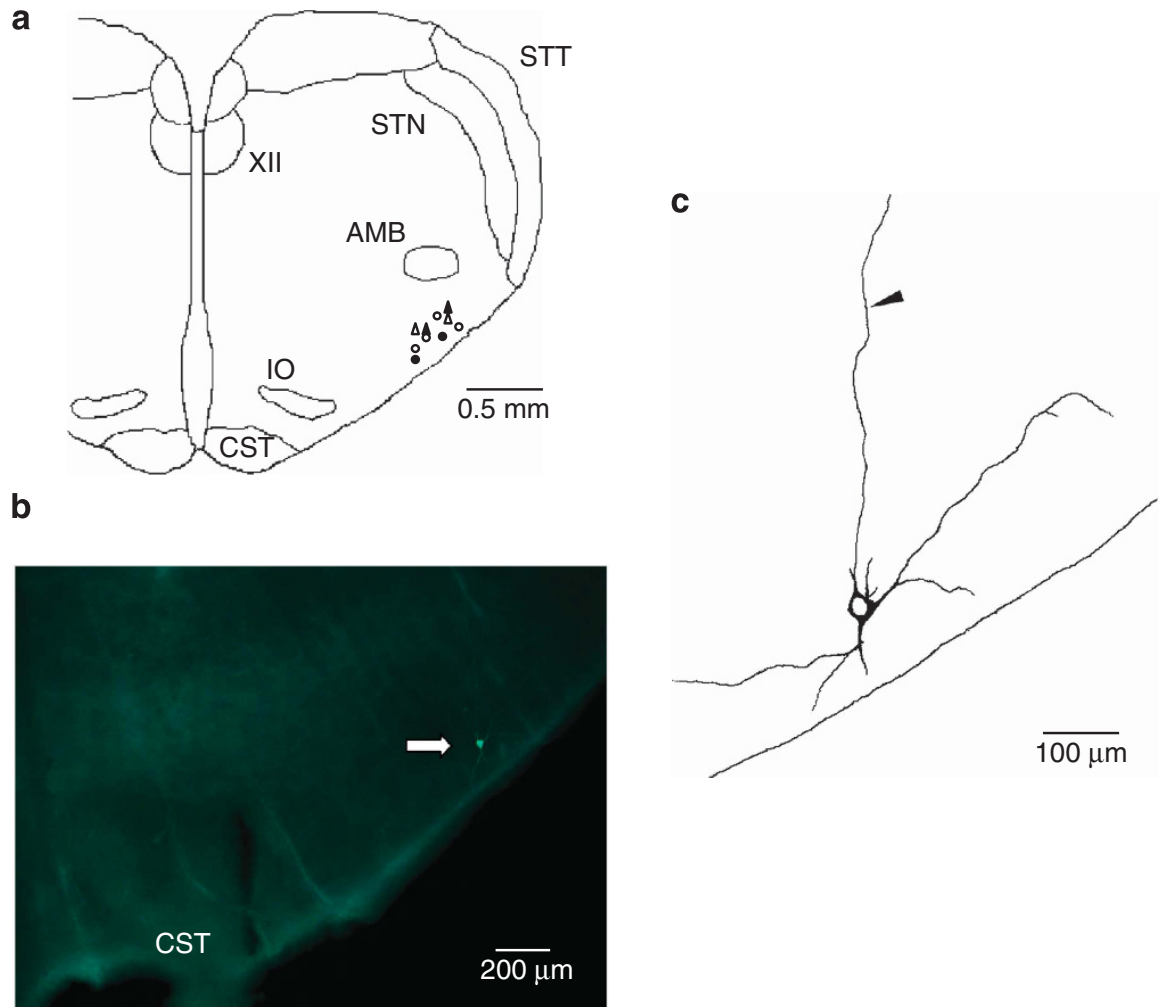

Figure 7 Microscopic examination of a recorded bulbospinal RVLM neuron in transverse section. The neuron is indicated in (b and $\mathbf{c})$ is the one in which the MP is shown in Figure 6b. (a) Cross-section of the medulla oblongata and 10 bulbospinal RVLM neurons examined histologically. ( $\bullet$ ) Regularly firing neurons; ( $O$ ) irregularly firing neurons; $(\boldsymbol{\Lambda})$ silent-type neurons; $(\Delta)$ burst-type neurons; AMB, nucleus ambiguous (rostral compact portion); CST, corticospinal tract; IO, inferior olivary nucleus; STN, spinal trigeminal nucleus; STT, spinal trigeminal tract; XII, hypoglossal nucleus. (b) A photograph of a neuron stained with Lucifer-Yellow at a magnification of $\times 100$. The arrow denotes the stained neuron. (c) Drawings of a Lucifer-Yellow-stained neuron. The arrowhead denotes the axon.

the 14 bulbospinal RVLM neurons depolarized during superfusion with aldosterone dissolved in a low- $\mathrm{Ca}^{2+}$, high- $\mathrm{Mg}^{2+}$ solution (Figure 2d, Table 1B). As most of the bulbospinal RVLM neurons were depolarized, aldosterone may activate those neurons directly.

Aldosterone has been shown to exert its effects through genomic or non-genomic action. ${ }^{28,29}$ Although in some previous studies aldosterone and eplerenone was infused into the intracerebroventricles, ${ }^{2,3,21}$ whether the responses were due to genomic action was not determined. In the present study, because the depolarization of the RVLM neurons occurred within a few minutes after starting aldosterone superfusion, the effects of aldosterone on the RVLM neurons were considered to be via a non-genomic action.

In three of the 19 bulbospinal RVLM neurons, aldosterone hyperpolarized their activity (Table 1A). Furthermore, four of the 14 bulbospinal RVLM neurons were hyperpolarized during superfusion with aldosterone dissolved in a low- $\mathrm{Ca}^{2+}$, high- $\mathrm{Mg}^{2+}$ solution (Table 1B). Although the mechanism is unknown, aldosterone may also suppress the activity of some of the bulbospinal RVLM neurons.

\section{Effect of eplerenone on bulbospinal neurons in the RVLM}

In 13 of the 15 bulbospinal RVLM neurons, eplerenone decreased RVLM neuronal activity (Figure 3a, Table 1A), and eight of the 10 bulbospinal RVLM neurons were hyperpolarized during superfusion with eplerenone dissolved in a low- $\mathrm{Ca}^{2+}$, high- $\mathrm{Mg}^{2+}$ solution (Figure 3c, Table 1B). These results suggest that eplerenone hyperpolarized the bulbospinal RVLM neurons' activity directly and that MRs exist in these neurons. In all four of the RVLM neurons that were hyperpolarized during eplerenone superfusion, the addition of aldosterone did not induce any obvious effects in the RVLM neurons (Figure 3d). These results suggest that eplerenone administered as a pretreatment bound to the MRs and suppressed the effects of aldosterone on the RVLM neurons.

In the brain, MRs have been reported to exist in hippocampal neurons and nuclei of the anterior hypothalamus, amygdale and brainstem. ${ }^{30}$ Gomez-Sanchez ${ }^{30}$ showed that intracerebroventricular infusion of inhibitors of steroidogenesis prevented the increase in blood pressure in salt-sensitive rats, and Felder ${ }^{31}$ found that activation of brain MRs increased sympathetic discharge. Westcott et al. ${ }^{5}$ showed that central blockade of aldosterone production and MRs prevented cardiac remodeling and dysfunction after myocardial infarction. However, the existence of MRs in the bulbospinal RVLM neurons remains undetermined. In this study, because the bulbospinal RVLM neurons depolarized or hyperpolarized during superfusion with aldosterone or eplerenone dissolved in a low- $\mathrm{Ca}^{2+}$, high- $\mathrm{Mg}^{2+}$ solution, we concluded that the RVLM neurons have MRs; moreover, MRs are responsible for aldosterone-induced RVLM depolarization.

In six of the 13 hyperpolarized RVLM neurons, an obvious decrease in EPSP amplitudes was shown during eplerenone superfusion (Figure 3b). The activity of the neurons (except CVLM neurons) that sent signals to the RVLM neurons was probably suppressed during eplerenone superfusion, or the CVLM neurons that sent signals to the bulbospinal RVLM neurons were activated. 
There were three cases in which the RVLM neurons were hyperpolarized during aldosterone superfusion (Table 1A), and we examined the effect of eplerenone on these neurons. In two cases, the bulbospinal RVLM neurons were hyperpolarized during aldosterone superfusion and depolarized during eplerenone superfusion (Figure 4a). Interestingly, eplerenone depolarized these neurons. Although the mechanism by which aldosterone suppressed the RVLM neurons is unknown, these results suggest that relative to aldosterone, eplerenone has the opposite effect on RVLM neurons.

\section{Effect of FAD286 on bulbospinal neurons in the RVLM}

The observation that eplerenone hyperpolarizes RVLM neurons suggests that some steroids are capable of stimulating MRs in RVLM neurons. In addition to aldosterone, glucocorticoids are also known to bind to MRs and activate them. ${ }^{32,33}$ Thus, to evaluate endogenously produced aldosterone, we performed an FAD286 superfusion. As a result, all five RVLM neurons became hyperpolarized (Figures 5Aa, b and B Table 1A), suggesting the existence of aldosterone in the brainstem, including the RVLM region. The duration needed for recovery of the MPs after cessation of the FAD286 superfusion was longer than $20 \mathrm{~min}$ (Figure 5b). These results suggest that some time is required to synthesize aldosterone. Furthermore, our finding that FAD286 dissolved in a low-Ca ${ }^{2+}$, high- $\mathrm{Mg}^{2+}$ solution caused hyperpolarized RVLM neurons (Figure 5c, Table 1B), suggests that aldosterone exists and is synthesized in the RVLM. In a previous study, Connell et al. ${ }^{1}$ showed that the central nervous system can produce aldosterone. Furthermore, using FAD286, Gomez-Sanchez et al. ${ }^{2}$ and Hung et al. ${ }^{6,7}$ proved that aldosterone is synthesized in the brain. Kumar et al. ${ }^{4}$ demonstrated the presence of aldosterone-related mRNA species in the RVLM. However, to our knowledge, no previous reports have shown any evidence that endogenous aldosterone has effects in the RVLM. Therefore, our study may be the first to suggest that endogenous aldosterone activates bulbospinal RVLM neurons.

\section{Effect of ENaC on bulbospinal neurons in the RVLM}

In the brain, $\mathrm{ENaC}$ has been reported to exist in the supraoptic nucleus, magnocellular paraventricular nucleus, hippocampus, choroid plexus and ependyma. ${ }^{22,34,35}$ Wang et al. ${ }^{36}$ showed the presence of $\mathrm{ENaC}$ in the choroid plexus and the ependyma of the anteroventral third ventricle, and reported that intracerebroventricular infusion of aldosterone increased blood pressure and intracerebroventricular infusion of benzamil blocked this increase. To our knowledge, there are no reports that have indicated the existence of ENaCs in bulbospinal RVLM neurons.

In the present study, we showed the effect of benzamil in the bulbospinal RVLM neurons. In 14 of the 18 neurons, the bulbospinal RVLM neurons were hyperpolarized during benzamil superfusion (Figure 6a, Table 1A). In 3 of the 14 hyperpolarized neurons, an obvious decrease in EPSP amplitudes was shown during benzamil superfusion (Figure 6a). The results suggest that activation by some of the neurons (except for the CVLM neurons), which were sending signals to the RVLM neurons, was suppressed during benzamil superfusion. To block the signals from other neurons that synapse with RVLM neurons, the brainstem-spinal preparations were superfused with a low- $\mathrm{Ca}^{2+}$, high- $\mathrm{Mg}^{2+}$ solution. During superfusion with benzamil dissolved in a low- $\mathrm{Ca}^{2+}$, high- $\mathrm{Mg}^{2+}$ solution, all of the 7 bulbospinal RVLM neurons were hyperpolarized (Figure $6 \mathrm{~b}$ and Table 1B). Aldosterone, added to a benzamil superfusion dissolved in a low- $\mathrm{Ca}^{2+}$, high- $\mathrm{Mg}^{2+}$ solution, did not depolarize the RVLM neurons (Figure 6b). These results strongly suggest that ENaCs are responsible for aldosterone-induced RVLM depolarization.

The mechanisms by which $\mathrm{ENaC}$ inhibition leads to suppressed RVLM neuron activity are unknown. Previous reports have shown the effect of benzamil. Benzamil injected into brain ventricles improved hypertension by releasing vasopresin ${ }^{37}$ or suppressing brain reninangiotensin system (RAS) mRNAs $^{38}$ by blocking brain sodium channels in salt-sensitive hypertension rats. Moreover, benzamil reportedly activates endothelial nitric oxide synthase (eNOS) and produces nitric oxide (NO) in rat mesenteric arteries by blocking ENaCs. ${ }^{26}$ In this study, these mechanisms may have influenced the MPs of the RVLM neurons in addition to blocking the inward sodium ion current.

The mechanisms underlying the sympathetic hyperactivity caused by aldosterone have not yet been established. According to previous reports, ${ }^{36,39}$ aldosterone in the brain causes ouabain production and oxidative stress, which in turn stimulates sympathetic outflow in the central nervous system. Our results suggest that aldosterone depolarized bulbospinal RVLM neurons directly and that ENaCs are involved in this action. The stimulation of RVLM neurons by aldosterone through ENaCs may be a pathway of sympathetic hyperactivity, in addition to the "ouabain or oxidative stress pathway" caused by aldosterone.

\section{Bulbospinal RVLM neurons can be classified into four types based} on their firing patterns

In this study, we found burst-type neurons, in addition to the three other types of neurons, which we previously reported. ${ }^{12}$ As these neurons had antidromic APs evoked by electrical stimulation in the intermediolateral cell column at the $\mathrm{Th}_{2}$ level, they participate in sympathetic nerve discharge. They also depolarized during aldosterone superfusion. The resting MPs of these burst-type neurons were more negative than those of regularly firing neurons.

Toney et al. ${ }^{40}$ reported the existence of burst-firing RVLM neurons that are barosensitive and respiratory rhythmic, using an extracellular recording method in vivo. In our study, the burst-type neurons in the RVLM may have been modulated by the respiratory network, but their firing patterns differed from those of the respiratory neurons ${ }^{14}$ recorded using whole-cell recordings from a preparation similar to ours.

In summary, aldosterone depolarized and eplerenone hyperpolarized the bulbospinal RVLM neurons directly, suggesting that MRs are involved in the depolarization of RVLM neurons. The result that FAD286 hyperpolarized the bulbospinal RVLM neurons directly suggests that aldosterone is synthesized in the RVLM. We demonstrated that benzamil hyperpolarized the bulbospinal RVLM neurons directly, and aldosterone had no effects during superfusion with benzamil dissolved in a low- $\mathrm{Ca}^{2+}$, high- $\mathrm{Mg}^{2+}$ solution. These data suggest that ENaCs are involved in aldosterone-induced depolarization of RVLM neurons.

Synthesized aldosterone in the RVLM may contribute to increased sympathetic nerve activity by activating the bulbospinal RVLM neurons through ENaCs, which leads to increased blood pressure. In the hypertensive state, increased aldosterone production may occur in the RVLM.

\footnotetext{
1 Connell JM, Davies E. The new biology of aldosterone. J Endocrinol 2005; 186: 1-20. 2 Gomez-Sanchez EP, Gomez-Sanchez CM, Plonczynski M, Gomez-Sanchez CE. Aldosterone synthesis in the brain contributes to Dahl salt-sensitive rat hypertension. Exp Physiol 2010; 95: 120-130.
} 
3 Zhang ZH, Yu Y, Kang YM, Wei SG, Felder RB. Aldosterone acts centrally to increase brain renin-angiotensin system activity and oxidative stress in normal rats. Am J Physiol Heart Circ Physiol 2008; 294: H1067-H1074.

4 Kumar NN, Goodchild AK, Li Q, Pilowsky PM. An aldosterone-related system in the ventrolateral medulla oblongata of spontaneously hypertensive and Wistar-Kyoto rats. Clin Exp Pharmacol Physiol 2006; 33: 71-75.

5 Westcott KV, Huang BS, Leenen FH. Brain renin-angiotensin-aldosterone system and ventricular remodeling after myocardial infarct: a review. Can J Physiol Pharmacol 2009; 87: 979-988.

6 Huang BS, White RA, Ahmad M, Tan J, Jeng AY, Leenen FH. Central infusion of aldosterone synthase inhibitor attenuates left ventricular dysfunction and remodelling in rats after myocardial infarction. Cardiovasc Res 2009; 81: 574-581.

7 Huang BS, White RA, Ahmad M, Jeng AY, Leenen FH. Central infusion of aldosterone synthase inhibitor prevents sympathetic hyperactivity and hypertension by central $\mathrm{Na}+$ in Wistar rats. Am J Physiol Regul Integr Comp Physiol 2008; 295: R166-R172.

8 Masuda T, Hirabara Y, Nakamura Y, Chishaki A, Tsuruhisa M, Miyakawa M, Honda K, Saito R, Sakamoto H, Kawata M, Takano Y. Aldosterone-sensitive nucleus tractus solitarius neurons regulate sensitivity of the baroreceptor reflex in high sodium-loaded rats. J Pharmacol Sci 2010; 112: 482-486.

9 Zhu DN, Xue LM, Li P. Cardiovascular effects of microinjection of corticoids and antagonists into the rostral ventrolateral medulla in rats. Blood Press 1995; 4: $55-62$.

10 Nakagaki T, Hirooka Y, Matsukawa R, Nishihara M, Nakano M, Ito K, Hoka S, Sunagawa K. Activation of mineralocorticoid receptors in the rostral ventrolateral medulla is involved in hypertensive mechanisms in stroke-prone spontaneously hypertensive rats. Hypertens Res 2012; 35: 470-476.

11 ligaya $\mathrm{K}$, Kumagai $\mathrm{H}$, Onimaru $\mathrm{H}$, Kawai $\mathrm{A}$, Oshima $\mathrm{N}$, Onami $\mathrm{T}$, Takimoto $\mathrm{C}$, Kamayachi T, Hayashi K, Saruta T, Itoh H. Novel axonal projection from the caudal end of the ventrolateral medulla to the intermediolateral cell column. Am J Physiol Regul Integr Comp Physiol 2007; 292: R927-R936.

12 Oshima N, Kumagai H, Kawai A, Sakata K, Matsuura T, Saruta T. Three types of putative presympathetic neurons in the rostral ventral medulla studied with rat brainstem-spinal cord preparation. Auton Neurosci 2000; 84: 40-49.

13 Onimaru $\mathrm{H}$, Homma I. Whole cell recording from respiratory neurons in the medulla of brainstem-spinal cord preparations isolated from newborn rats. Pflugers Arch 1992 420: 399-406.

14 Onimaru H, Arata A, Homma I. Neuronal mechanisms of respiratory rhythm generation: an approach using in vitro preparation. Jpn J Physiol 1997; 47: 385-403.

15 Lin HH, Wu SY, Lai CC, Dun NJ. GABA- and glycine-mediated inhibitory postsynaptic potentials in neonatal rat rostral ventrolateral medulla neurons in vitro. Neuroscience 1998; 82: 429-442.

16 Matsuura T, Kumagai H, Kawai A, Onimaru H, Imai M, Oshima N, Sakata K, Saruta T. Rostral ventrolateral medulla neurons of neonatal Wistar-Kyoto and spontaneously hypertensive rats. Hypertension 2002; 40: 560-565.

17 Matsuura T, Kumagai H, Onimaru H, Kawai A, ligaya K, Onami T, Sakata K, Oshima N, Sugaya T, Saruta T. Electrophysiological properties of rostral ventrolateral medulla neurons in angiotensin II la receptor knockout mice. Hypertension 2005; 46 349-354.

18 Deuchars SA, Morrison SF, Gilbey MP. Medullary-evoked EPSPS in neonatal rat sympathetic preganglionic neurons in vitro. J Physiol 1995; 487: 453-463.

19 Oshima N, Kumagai H, Onimaru H, Kawai A, Pilowsky PM, ligaya K, Takimoto C, Hayashi K, Saruta T, Itoh $\mathrm{H}$. Monosynaptic excitatory connection from the rostral ventrolateral medulla to sympathetic preganglionic neurons revealed by simultaneous recordings. Hypertens Res 2008; 31: 1445-1454.

20 Oshima N, Kumagai H, ligaya K, Onimaru H, Kawai A, Nishida Y, Saruta T, Itoh H. Baro-excited neurons in the caudal ventrolateral medulla (CVLM) recorded using the whole-cell patch-clamp technique. Hypertens Res 2012; 35: 500-506.
21 Ito K, Hirooka Y, Sunagawa K. Blockade of mineralocorticoid receptors improves saltinduced left-ventricular systolic dysfunction through attenuation of enhanced sympathetic drive in mice with pressure overload. J Hypertens 2010; 28: 1449-1458.

22 Wang HW, Amin MS, El-Shahat E, Huang BS, Tuana BS, Leenen FH. Effects of central sodium on epithelial sodium channels in rat brain. Am J Physiol Regul Integr Comp Physiol 2010; 299: R222-R233.

23 Ishizawa K, Izawa Y, Ito H, Miki C, Miyata K, Fujita Y, Kanematsu Y, Tsuchiya K, Tamaki T, Nishiyama A, Yoshizumi M. Aldosterone stimulates vascular smooth muscle cell proliferation via big mitogen-activated protein kinase 1 activation. Hypertension 2005; 46: 1046-1052.

24 Nguyen Dinh Cat A, Briones AM, Callera GE, Yogi A, He Y, Montezano AC, Touyz RM. Adipocyte-derived factors regulate vascular smooth muscle cells through mineralocorticoid and glucocorticoid receptors. Hypertension 2011; 58: 479-488.

25 Quesnell RR, Han X, Schultz BD. Glucocorticoids stimulate ENaC upregulation in bovine mammary epithelium. Am J Physiol Cell Physiol 2007; 292: C1739-C1745.

26 Pérez FR, Venegas F, González M, Andrés S, Vallejos C, Riquelme G, Sierralta J, Michea L. Endothelial epithelial sodium channel inhibition activates endothelial nitric oxide synthase via phosphoinositide 3-kinase/Akt in small-diameter mesenteric arteries. Hypertension 2009; 53: 1000-1007.

27 Haas HL, Jefferys JG. Low-calcium field burst discharges of CA1 pyramidal neurones in rat hippocampal slices. J Physiol 1984; 354: 185-201.

28 Dooley R, Harvey BJ, Thomas W. Non-genomic actions of aldosterone: From receptors and signals to membrane targets. Mol Cell Endocrinol 2012; 350: 223-234.

29 Zhang ZH, Yu Y, Wei SG, Felder RB. Aldosterone-induced brain MAPK signaling and sympathetic excitation are angiotensin II type-1 receptor dependent. Am J Physiol Heart Circ Physiol 2011; 302: H742-H751.

30 Gomez-Sanchez EP. The mammalian mineralocorticoid receptor: tying down a promiscuous receptor. Exp Physiol 2010; 95: 13-18.

31 Felder RB. Mineralocorticoid receptors, inflammation and sympathetic drive in a rat model of systolic heart failure. Exp Physiol 2010; 95: 19-25. Review.

32 Fuller PJ, Yao Y, Yang J, Young MJ. Mechanisms of ligand specificity of the mineralocorticoid receptor. J Endocrinol 2012; 213: 15-24.

33 de Kloet ER, Van Acker SA, Sibug RM, Oitzl MS, Meijer OC, Rahmouni K, de Jong W. Brain mineralocorticoid receptors and centrally regulated functions. Kidney Int 2000; 57: 1329-1336.

34 Amin MS, Wang HW, Reza E, Whitman SC, Tuana BS, Leenen FH. Distribution of epithelial sodium channels and mineralocorticoid receptors in cardiovascular regulatory centers in rat brain. Am J Physiol Regul Integr Comp Physiol 2005; 289: R1787-R1797.

35 Amin MS, Reza E, Wang H, Leenen FH. Sodium transport in the choroid plexus and salt-sensitive hypertension. Hypertension 2009; 54: 860-867.

36 Wang H, Huang BS, Leenen FH. Brain sodium channels and ouabainlike compounds mediate central aldosterone-induced hypertension. Am J Physiol Heart Circ Physiol 2003; 285: H2516-H2523.

37 Nishimura M, Ohtsuka K, Nanbu A, Takahashi H, Yoshimura M. Benzamil blockade of brain $\mathrm{Na}+$ channels averts $\mathrm{Na}(+)$-induced hypertension in rats. Am J Physiol 1998; 274: R635-R644.

38 Nishimura M, Ohtsuka K, Iwai N, Takahashi H, Yoshimura M. Regulation of brain reninangiotensin system by benzamil-blockable sodium channels. Am J Physiol 1999; 276: R1416-R1424

39 Takahashi H, Yoshika M, Komiyama Y, Nishimura M. The central mechanism underlying hypertension: a review of the roles of sodium ions, epithelial sodium channels, the renin-angiotensin-aldosterone system, oxidative stress and endogenous digitalis in the brain. Hypertens Res 2011; 34: 1147-1160.

40 Toney GM, Pedrino GR, Fink GD, Osborn JW. Does enhanced respiratory-sympathetic coupling contribute to peripheral neural mechanisms of angiotensin II-salt hypertension? Exp Physiol 2010; 95: 587-594. 